\title{
Muscle cramps in the calf as presenting symptom of sarcoidosis
}

\author{
M Janssen, B A C Dijkmans, F Eulderink
}

\author{
Department of \\ Rheumatology, \\ University Hospital \\ Leiden, The Netherlands \\ $M$ Janssen \\ B A C Dijkmans \\ Department of \\ Pathology, University \\ Hospital, Leiden, \\ The Netherlands \\ F Eulderink \\ Correspondence to: \\ Dr M Janssen, \\ University Hospital, \\ Department of \\ Rheumatology, Building 1 \\ C2-Q, PO Box 9600 , \\ 2300 RC Leiden, \\ The Netherlands. \\ Accepted for publication \\ 14 February 1990
}

\begin{abstract}
A patient is described, who presented with pain in the calf due to a palpable nodule as the presenting symptom of sarcoidosis. The patient was treated with rest and diclofenac, followed by intralesional injections with triamcinolone hexacetonide and became free from pain.
\end{abstract}

Sarcoidosis is a multisystem granulomatous disorder of uncertain cause. Muscles are affected in 50 to $80 \%$ of patients with this disease. ${ }^{1}$ The muscle disease is usually asymptomatic but may be associated with chronic weakness, myalgia, pseudohypertrophy, and palpable nodules in the muscle. ${ }^{2}$ The last is the least common form of symptomatic sarcoid muscle disease, and may cause pain and stiffness with cramps. ${ }^{2}$ We describe a patient with pain in the calf due to a palpable nodule as the presenting symptom of sarcoidosis.

\section{Case report}

A 63 year old white woman visited our outpatient clinic in June 1987 complaining of several days of cramping pain of sudden onset in the left calf. Because of the pain she was unable to stand flat on her left foot, and the pain increased with walking. In addition, she had constant pain in the left calf at night and

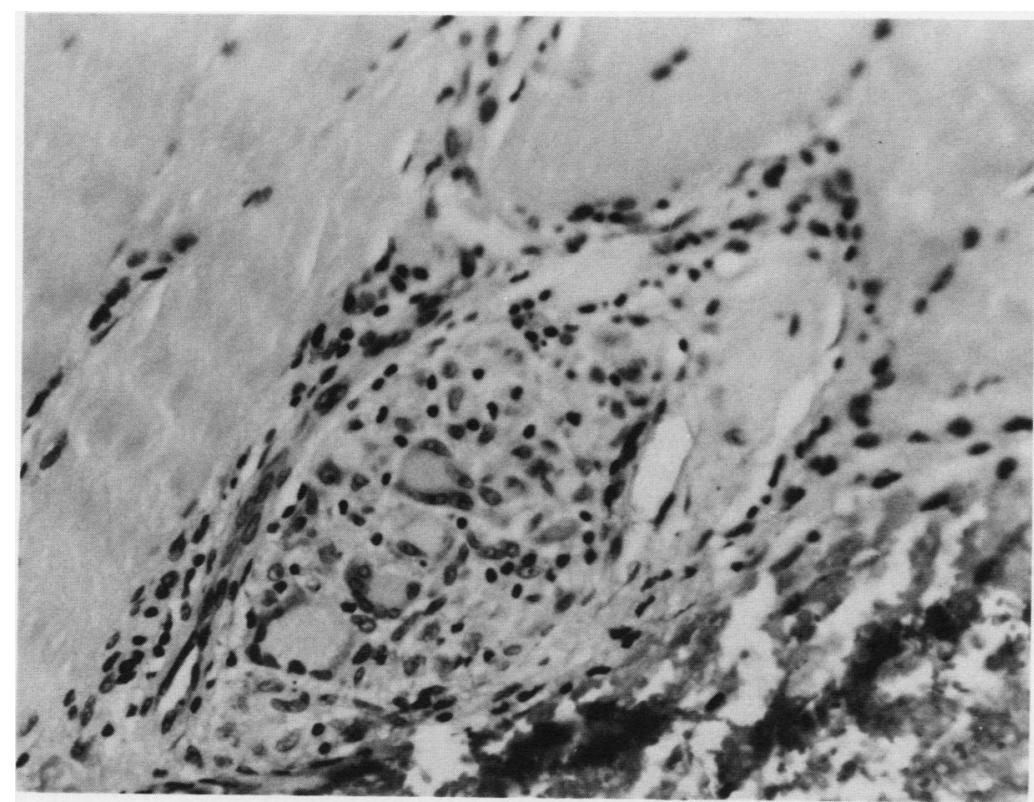

Non-necrotising epithelioid cell granuloma surrounded by muscle fibres. Three multinucleated giant cells of the Langhans type are visible in the granuloma. (Hacmatoxylin and eosin.) stiffness in the morning. Otherwise she had no complaints and felt healthy.

On examination no abnormalities were found except for a painful nodule $2 \mathrm{~cm}$ in diameter in the lateral border of the left gastrocnemius muscle at the junction with the Achilles tendon.

The erythrocyte sedimentation rate was $28 \mathrm{~mm}$ (Westergren) in the first hour, haemoglobin $119 \mathrm{~g} / \mathrm{l}$, and white blood cell count $6 \times 10^{9} / 1$ with a normal differential. The WaalerRose and latex fixation tests were negative and antinuclear factors absent. Liver, kidney, and thyroid functions were normal, as was the serum calcium. The creatine kinase was normal. The lysozyme was $312 \%$ (normal 55-195\%). The concentration of angiotensin converting enzyme was slightly raised at $70 \mu \mathrm{mol} / \mathrm{l}$ (normal 23-67 $\mu \mathrm{mol} / \mathrm{l}$ ). Arterial and venous ultrasound investigation of the legs was normal. The chest radiograph and pulmonary function tests were normal. Echography disclosed a hypoechogenic structure in the left calf of $3 \mathrm{~cm}$ in diameter. A biopsy specimen was taken from this structure, which showed several non-necrotising granulomas between the muscle fibres and in adjacent fat tissue with epithelioid cells, multinucleated giant cells, and lymphocytes, typical of sarcoidosis (figure). The Ziehl-Neelsen stain was negative; no bacteria or yeasts could be cultured from this material.

Based on the findings of the biopsy, the diagnosis sarcoidosis was made and the patient was treated with rest and diclofenac. This symptomatic treatment was only partially effective, but after intralesional injections with triamcinolone hexacetonide the patient became completely free from pain. About one month after her first visit a repeat chest radiograph showed bilateral hilar lymphadenopathy. The erythrocyte sedimentation rate returned to normal after three months, the hilar lymphadenopathy and the angiotensin converting enzyme concentration after about two years. During the course of the disease no abnormalities of laboratory tests developed.

\section{Discussion}

The main conclusion to be drawn from this case history is that muscle cramps in the calf due to a nodule may be the presenting symptom of sarcoidosis.

Muscle disease in sarcoidosis may be asymptomatic or symptomatic. Patients with symptoms typically have a chronic, slowly progressive muscle disease, often with wasting, and closely resembling chronic polymyositis or muscular dystrophy. ${ }^{4}$ The major presenting feature of 
symptomatic muscle disease is weakness, occurring in three quarters of patients, ${ }^{2}$ while one fifth of cases present with myalgia only. ${ }^{2}$ Other symptomatic forms include palpable muscle nodules and acute myositis. Presentation with tender palpable nodules is extremely rare. ${ }^{25}$

In our patient a biopsy specimen was taken from the affected tissue. The presence of noncaseating epithelioid cell granulomas made the diagnosis sarcoidosis probable. Other conditions associated with granulomas, such as tuberculosis, fungal infections, and connective tissue disease, rarely produce skeletal muscle granulomas but should be considered in the differential diagnosis. In our patient we found no signs or symptoms of these diseases and the bihilar lymphadenopathy, shown by the second chest radiograph, and the increased concentration of angiotensin converting enzyme gave further support to the diagnosis of sarcoidosis. Some aspects of the developing lymphadenopathy have to be emphasised. Firstly, there is no agreement as to whether skeletal muscle sarcoidosis might occur in the absence of other organ disease $^{2}$; in this case the muscle sarcoidose seemed to be the first sign of a multisystem disorder. Secondly, there is some discussion about whether it is worthwhile repeating the chest radiograph after several weeks when a diagnosis of sarcoidosis is suspected and the first radiograph is normal. Results with our patient show that this is worthwhile.

Treatment with corticosteroids has been advocated for myopathy in sarcoidosis. ${ }^{2}$ The general condition of our patient did not require treatment with corticosteroids, however, and treatment with non-steroidal anti-inflammatory drugs was started, only to be replaced later by intralesional corticosteroid. This treatment, though unusual, was successful and avoided the use of systemic corticosteroids and their possibly adverse reactions.

In conclusion, the doctor should be aware of the possibility of sarcoid myopathy in a patient with unexplained muscle pain. A careful palpation of the affected muscle is indicated.

We would like to thank Dr J D Macfarlane for correcting the English text.

1 Silverstein A, Siltzbach L E. Muscle involvement in sarcoidosis. Arch Neurol 1969; 21: 235-41.

2 Wolfe S M, Pinals R S, Aelion J A, Goodman R E. Myopathy in sarcoidosis: clinical and pathologic study of four cases and review of the literature. Semin Arthritis Rheum 1987; 16: $300-6$.

3 Matteson E L, Michet C J. Sarcoid myositis with pseudohypertrophy. Am $\mathcal{Y}$ Med 1989; 87: 240.

4 Alpert J N, Groff A E, Bastian F O, Blum M A. Acute polymyositis caused by sarcoidosis: report of a case and review of the literature. Mt Sinai $\mathcal{f}$ Med $(N Y)$ 1979; 46 ; 486-8.

5 Bates G S, Walsh J M. Boeck's sarcoid, observations on seven patients, and one autopsy. Ann Intern Med 1948; 29: 306-17. 